KfK 2464

Juli 1978

\title{
Nuclear Charge Radii and Nuclear Moments of Neutron Deficient Ba Isotopes from High Resolution Laser Spectroscopy
}

\author{
G. Nowicki, K. Bekk, S. Göring \\ A. Hanser, H. Rebel, G. Schatz \\ Institut für Angewandte Kernphysik
}

Kernforschungszentrum Karlsruhe 
Als Manuskript vervielfältigt

Für diesen Bericht behaiten wir uns alle Rechte vor

KERNFORSCHUNGSZENTRUM KARLSRUHE GMBH 


\section{KERNFORSCHUNGSZENTRUM KARLSRUHE}

Institut für Angewandte Kernphysik

KfK 2464

NUCLEAR CHARGE RADII AND NUCLEAR MOMENTS OF NEUTRON DEFICIENT Ba ISOTOPES FROM HIGH RESOLUTION LASER SPECTROSCOPY

G. Nowicki, K. Bekk, S. Göring,

A. Hanser, H. Rebel, and G. Schatz 



\section{Abstract}

Isotope shifts and hyperfine structure of the BaI $6 s^{2}{ }^{1} s_{0}-6 s 6 p{ }^{1} P_{1}$ transitions $(\lambda=553.6 \mathrm{~nm})$ in neutron deficient Ba nuclides $(\mathrm{N}<82)$ have been investigated by observing the resonance fluorescence in a well collimated atomic beam with a high resolution tunable CW dye laser. The experiments aimed at information on nuclear charge radii and on nuclear moments. Results are presented for the radioactive isotopes and isomers $133 \mathrm{~g}_{\mathrm{Ba}}, 133 \mathrm{~m}_{\mathrm{Ba}},{ }^{131} \mathrm{Ba}$, ${ }^{128} \mathrm{Ba}$, in addition to remeasurements of all stable $\mathrm{Ba}$ nuclides. The extracted values of $\delta\left\langle r^{2}\right\rangle$, the observed odd-even staggering and the nuclear moments are discussed in the light of other theoretical and experimental nuclear structure studies of the region $50 \leq(Z, N) \leq 82$.

Ladungsradien und Kernmomente neutronenarmer Barium-Isotope aus laserspektroskopischen Experimenten hoher Auflösung

Isotopieverschiebung und Hyperfeinstruktur des BaI $6 s^{2}{ }^{1} s_{0}-6 s 6 p{ }^{1} p_{1}$ Übergangs $(\lambda=553,6 \mathrm{~nm})$ in neutronenarmen Ba-Nukliden $(\mathrm{N}<82)$ wurden untersucht. durch Beobachtung der Resonanzfluoreszenz nach Anregung der Ba-Atome eines gut kollimierten Atomstrahls mittels eines hochauflösenden durchstimmbaren $\mathrm{CW}$-Lasers. Die Experimente zielten auf Informationen über nukleare Ladungsradien und Kernmomente. Zusätzlich zu Nachmessungen an allen stabilen Ba-Isotopen wurden die radioaktiven Isotope ${ }^{128} \mathrm{Ba},{ }^{131} \mathrm{Ba},{ }^{133 g_{\mathrm{Ba}}}$ und das Isomer ${ }^{133 \mathrm{~m}} \mathrm{Ba}$ untesucht. Die gewonnenen Resultate über $\delta\left\langle r^{2}\right\rangle$, das beobachtete "odd-even staggering" und die Kernmomente werden im Lichte anderer Ergebnisse über die Kernstruktur im Bereich $50 \leq(\mathrm{N}, \mathrm{Z}) \leq 82$ diskutiert. 


\section{Introduction}

The investigation of nuclear effects in atomic transitions by optical spectroscopy is one of the classical tools for determining basic nuclear quantities such as the spin, the charge distribution, the magnetic dipole and electric quadrupole moment. The recent review article of Arimondo et al. ${ }^{1}$ compiling the experimental methods and, particularly, the results for alkali atoms demonstrates the potential, the detailed aspects and the richness of the data collected by this specific type of nuclear spectroscopy. In recent years the development of extraordinary light sources such as tunable lasers has led to new spectroscopic methods ${ }^{2}$ of very high resolution (overcoming the limits due to the Doppler broadening of optical lines) and a fascinating gain of sensitivity. This has made it possible to apply optical spectroscopy to very small numbers of atoms and thus to extend the experimental studies to rather short-lived radioactive isotopes. In fact, atomic spectroscopy provides the only experimentally practicable method for determining rms charge radii of nuclei far off the stability line. The knowledge or spin, moments and charge radii along isotopicchains, including the unstable nuclei, seems to be indispensable, however, for a systematic understanding of nuclear structure. An impressive example of the interesting insight obtained by such studies is given by the exciting work on the Hg isotopes ${ }^{3}$.

In the present paper we report on measurements of isotope shifts and hyperfine structure of neutron deficient $\mathrm{Ba}$ isotopes with neutron number below 82 . The experiments are based on the observation of the resonance fluorescence of the atomic $6 s^{2}{ }^{1} S_{O}-6 s 6 p{ }^{1} P_{1}$ transition in Ba I when excited in an atomic beam by a high resolution tunable dye laser. Systematic investigations 4 of the level schemes and transition probabilities of neutron deficient nuclei in the mass region with $50 \leq(\mathrm{Z}, \mathrm{N}) \leq 82$ have revealed an increasing nuclear 
deformation with decreasing neutron number. A very illustrative picture of nuclear shapes of even-even nuclei in this region of the isotopic chart is presented by the collective energy surfaces ${ }^{5}$ derived from the analysis of the decay properties. Experimental knowledge of the ground state properties of the unstable nuclei, however, is very scarce. The rms charge radii of stable Ba nuclei have been investigated by electron scattering ${ }^{6}$, by measuring muonic ${ }^{7}$ and electronic $x$-rays ${ }^{8}$. The results of optical isotope shift measurements using classical spectroscopic methods ${ }^{9}$ are affected by rather large errors. Our present experiments aimed to extend the available information to unstable neutron deficient nuclei and to an improvement of the accuracy of known data on the stable isotopes, thus finally presenting a coherent set of results for the Ba nuclides. Our results on the stable Ba nuclei are in good agreement with recent laser spectroscopic measurements of the oxford group 10 as well as with results from level-crossing studies ${ }^{11}$. Classical methods of optical spectroscopy have been applied to the radioactive nuclides $14 \mathrm{O}_{\mathrm{Ba}}$

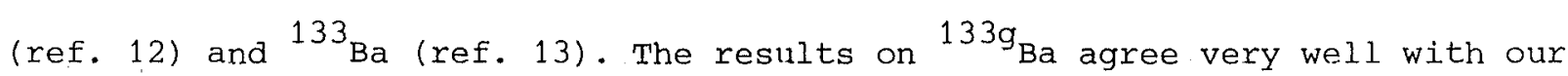
data. The very precise values ${ }^{14,15}$ of the magnetic dipole and electric quadrupole moments of the stable nuclei $135,137 \mathrm{Ba}$ are used as a calibration when extracting nuclear moments from the hyperfine structure in the spectra of neutron deficient isotopes. Part of our experimental results have been published previously 16 .

We discuss the results in terms of changes in charge rms radii and in nuclear deformation. With decreasing neutron number a large mean square deformation $\left\langle\beta^{2}\right\rangle$ is required to explain the extracted $\delta\left\langle r^{2}\right\rangle$ values. Comparing with the smaller values of the deformation parameters derived from measured $\mathrm{B}\left(\mathrm{E} 2, \mathrm{O}^{+} \rightarrow 2^{+}\right)$values the so called "isotope shift discrepancy" 17,32 is evident indicating a monotonic variation of the compressibility of the A nucleon system. 


\section{Experimentel set-up}

\subsection{Principle}

The experimental set-up which is schematically shown in fig. 1 consists of an apparatus producing a well collimated atomic beam of the radioactive atoms and a second reference beam operated with a stable isotope of $\mathrm{Ba}$. The Ba I

resonance transitions $6 s^{2}{ }^{1} s_{O}-6 s 6 p{ }^{1} P_{1}$ are induced by two tunable high resolution $\mathrm{CW}$ dye lasers in single mode operation. One dye laser is locked to the transition frequency of the stable $\mathrm{Ba}$ isotope in the reference atomic beam and provides an optical reference frequency. The frequency of the other laser is tuned through the resonance transition and excites fluorescence of

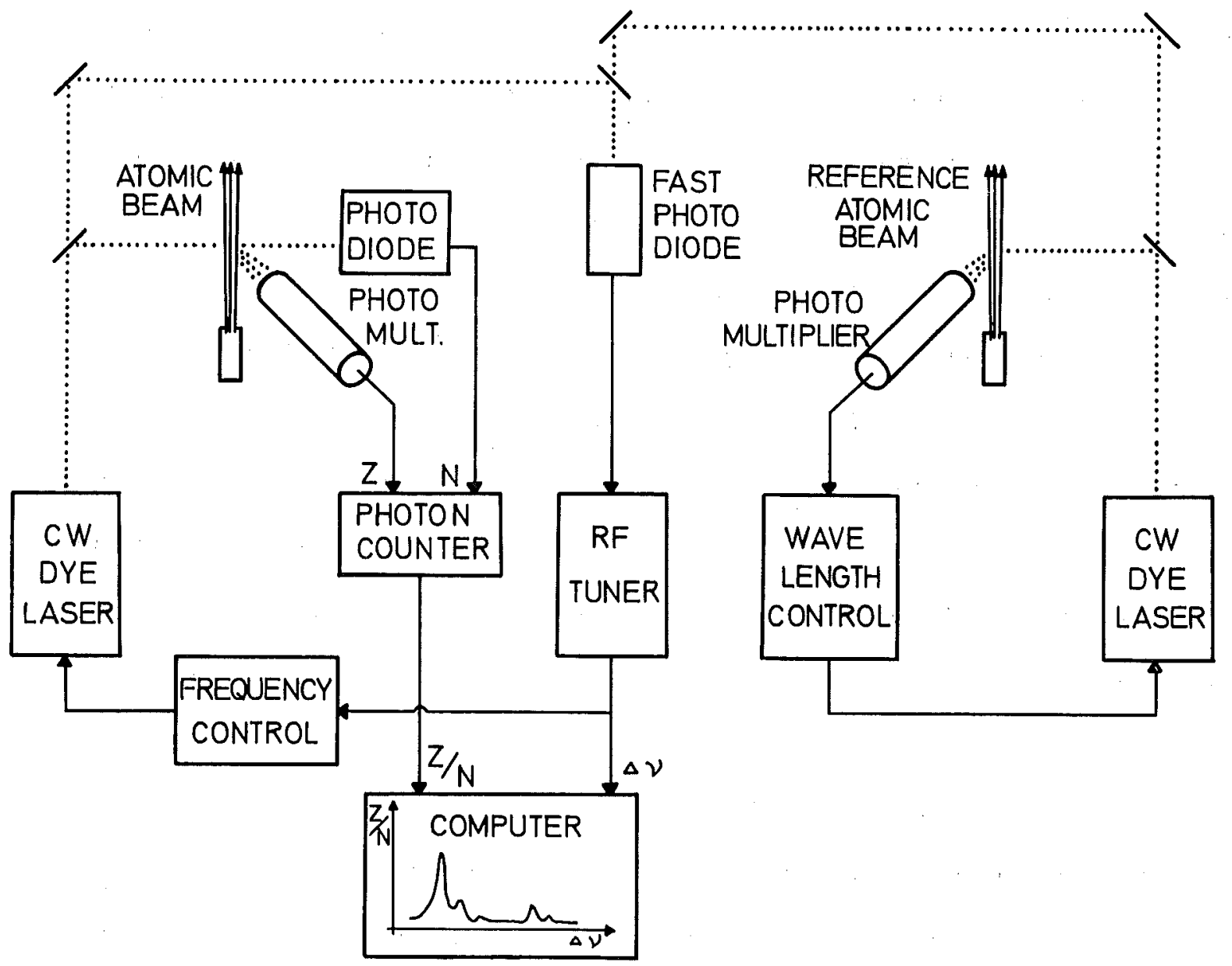

Fig. 1: Schematic view of the experimental set-up. The two dye lasers are pumped by one $\mathrm{Ar}^{+}$laser not shown in the figure. 
the unstable $\mathrm{Ba}$ atoms. It is controlled by stabilizing the difference of the two laser frequencies. This is achieved by mixing the light of the two laser beams on a fast photodiode and by comparing the difference frequency with the output signal of a RF generator. When tuning the RF generator the frequency of the second dye laser is shifted by the same amount. The fluorescence intensity is measured by a photomultiplier operated in photon counting mode.

\section{2 Dye lasers}

The $\mathrm{CW}$ dye lasers with folded resonators of the Kogelnik type 18 are built up according to a design of Hartig and walther ${ }^{19}$, with some modifications concerning the dye cell. With a dye solution of rhodamin $504 \cdot(35 \mathrm{mg} / 1)$ in water $(75 \%)$ and methanol $(25 \%)$ and some drops of COT laser emission ranges from $\lambda=548 \mathrm{~nm}$ to $\lambda=610 \mathrm{~nm}$ when pumped with the green $\mathrm{Ar}^{+}$laser line $(\lambda=514.4 \mathrm{~nm})$. At the $\mathrm{Ba} I$ resonance wave length $\lambda=553.55 \mathrm{~nm}$ the pump threshold is about $650 \mathrm{~mW}$ without intra cavity etalons. Single mode operation is achieved with two solid etalons; one with $4 \mathrm{~mm}$ thickness coated to $35 \%$ reflexion and an uncoated one of either 1,2 or $3 \mathrm{~mm}$. A pump power of $1.2 \mathrm{~W}$ is converted to $10 \mathrm{~mW}$ single mode output power at $\lambda=553.6 \mathrm{~nm}$, tunable in a range of $200 \mathrm{MHz}$ without tilting the etalons. Fine tuning of the dye laser frequency is done by piezoelectric driving of the output mirror of the laser resonator. The free running frequency stability is better than $10 \mathrm{MHz}$ (FHWM) for an observation time of some seconds. Piezoelectric movement of the spherical end mirror is used to lock the laser to a reference frequency.

\subsection{The optical reference frequency}

Very precise measurements of optical frequencies may be accomplished by a heterodyne technique if an adequate reference frequency is available differing by less than $5 \mathrm{GHz}$ from the frequency to be determined. The limitation is a consequence of the finite rise time (100 ps) of fast photodiodes presently 
available. The small isotope shifts $(<100 \mathrm{MHz})$ in the $\mathrm{Ba}$ I resonance lines of neighboring $\mathrm{Ba}$ isotopes permit the use of the resonance transitions of stable Ba isotopes $\left({ }^{136} \mathrm{Ba}\right.$ or $\left.{ }^{138} \mathrm{Ba}\right)$ as frequency standards. A CW dye laser locked to such a frequency serves as an optical reference oscillator in our experiment. A feedbacksignal for frequency locking is derived from the fluorescence signal, normally by differentiating this frequency dependent response signal by wobbling the laser frequency. An alternative procedure uses a reference value on the flank of the response signal. Thereby difficulties arise from intensity instabilities of the laser or from uncertainties in determining the reference frequency in an exact way.

In order to overcome these difficulties we introduced a different method using position sensitive detection of the fluorescence spot (see fig. 2). In a divergent atomic beam narrow banded laser light tuned to the resonance produces

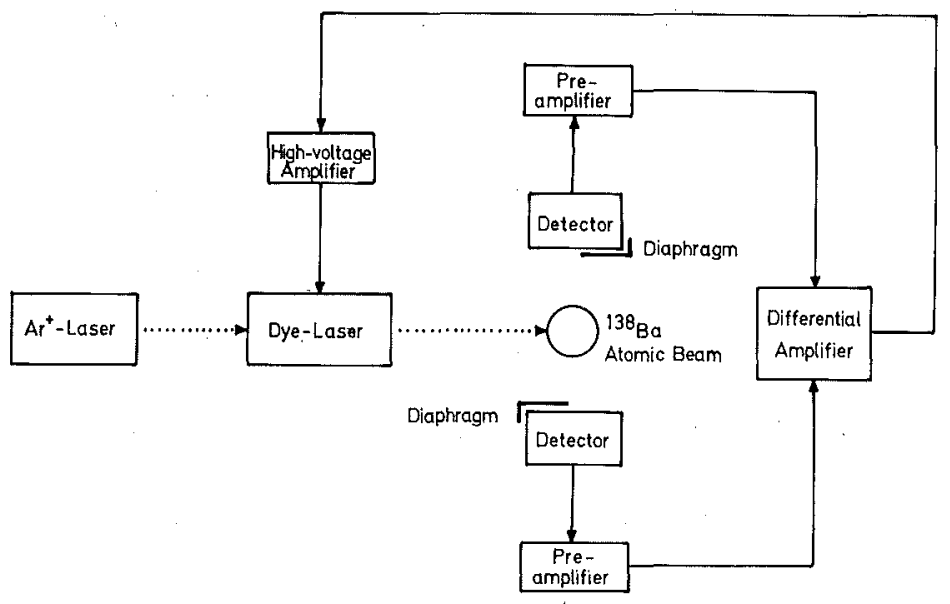

Fig. 2: The wavelength control system for the reference laser.

a very small fluorescence spot the position of which depends on the laser frequency due to the Doppler profile of the atomic beam. Decreasing the frequency of the laser light shifts the fluorescence spot into the laser beam direction. The fluorescence spot is imaged by aspherical lenses onto the photocathodes of two identical photomultipliers. Appropriate diaphragms guarantee that one photomultiplier looks only to the upstream side, the other one only to the downstream side of the laser beam. The difference current of both multipliers provides an error signal which is nearly independent of laser intensity fluctuations in the neighborhood of the reference frequency. The error 
signal is processed by a booster amplifier (HP 6826 A) driving the piezoelectric drive of the spherical end mirror of the laser. The range of control depends on the area seen by the multipliers and the thermal velocity of the atoms in the atomic beam. In our case it amounts to $\pm 60 \mathrm{MHz}$. The frequency stability obtained is better than $5 \mathrm{MHz}$ (FWHM).

\subsection{The difference frequency control}

The frequency of the laser beam exciting the resonance fluorescence of the unstable atoms has to be scanned relative to the optical reference frequency provided by the other laser. For this, part of the two laser beams are mixed on a fast photodiode (Spectra Physics Model 403) the photo current of which is modulated with the difference frequency of the two light beams. A RF-spectrum analyser (Hewlett Packard Model 8555) analyses the frequency of the diode signal. By woobling the center frequency of the RF-spectrum analyser a phase sensitive detector (lock-in-amplifier) converts the amplitude from the analyser into a signal that can be used for locking the second laser. The wobble frequency is typically $2 \mathrm{kHz}$ and the frequency stroke $\pm 10 \mathrm{MHz}$. By scanning the center frequency of the RF-spectrum analyser slowly (ca. $1 \mathrm{MHz} / \mathrm{s}$ ) the laser frequency is tuned relative to the fixed frequency of the reference laser. In order to decouple the fast frequency locking (with a small stroke) and the slow scanning with a large stroke, frequency locking uses the small. spherical end mirror while the tuning is done with the flat output coupler.

\subsection{The atomic beam apparatus}

One of the limiting experimental problems in optical spectroscopy of atomic transitions of radioactive isotopes is the rather small amount of material produced via nuclear reactions. In our cases, depending on the particular life-time and production process, at most some $10^{4}$ atoms per second can be expected in a well collimated atomic'beam. In order to avoid pumping of 
various hyperfine components of odd-A-isotopes while crossing the exciting laser beam, we can only profit from multiple excitation of a single atom for the even isotopes. In particular, multiple photon correlation techniques 20 do not improve the situation for odd isotopes. This requires a very low background and a highly efficient detection system in addition to an economic handling of the small number of radioactive atoms. These requirements indicate the design criteria for the apparatus used which is shown schematically in fig. 3. The top region of the oven cell consists of three exit channels $10.7 \mathrm{~mm}$ in diameter and with a length of $8 \mathrm{~mm}$ ) arranged in a plane perpendicular to the laser beam. Such a set up increases the efficiency compared to an arrangement with a single hole in the top of the oven. The oven material is molybdenum. An adjustable slit at $90 \mathrm{~mm}$ distance from the oven defines the beam divergence. In nearly all cases a slit width of $0.7 \mathrm{~mm}$ was chosen giving rise to a Doppler broadening of the resonance line of about $8 \mathrm{MHz}$. A small interchangeable $\mathrm{Al}$ sheet (with a hole for the central part of the atomic beam) catches most of the diverging atomic beam intensity and can be brought into a position where the collected $\gamma$-ray activity is measured by a Ge(Li) detector, thus monitoring the intensity of the atomic beam. Two glass lenses and a spherical mirror in the configuration shown in fig. 4 collect the resonance light quanta which are counted by a photomultiplier (EMI 9558 A) operated in photon counting mode. The light collection system covers a solid angle of $0.22 \cdot 4 \pi$. The efficiency of the photomultiplier is $16 \%$ at a wave length of $550 \mathrm{~nm}$. When some further light losses are accounted for this results in an overall photon detection efficiency of about $2 \%$

Background originates from scattered laser light, scattered incandescent light of the oven, from the dark current of the multiplier and from $\gamma$-radioactivity of the sample. Laser light background could be reduced very effectively by imaging only the small intersecting region of the atomic and the laser beams 


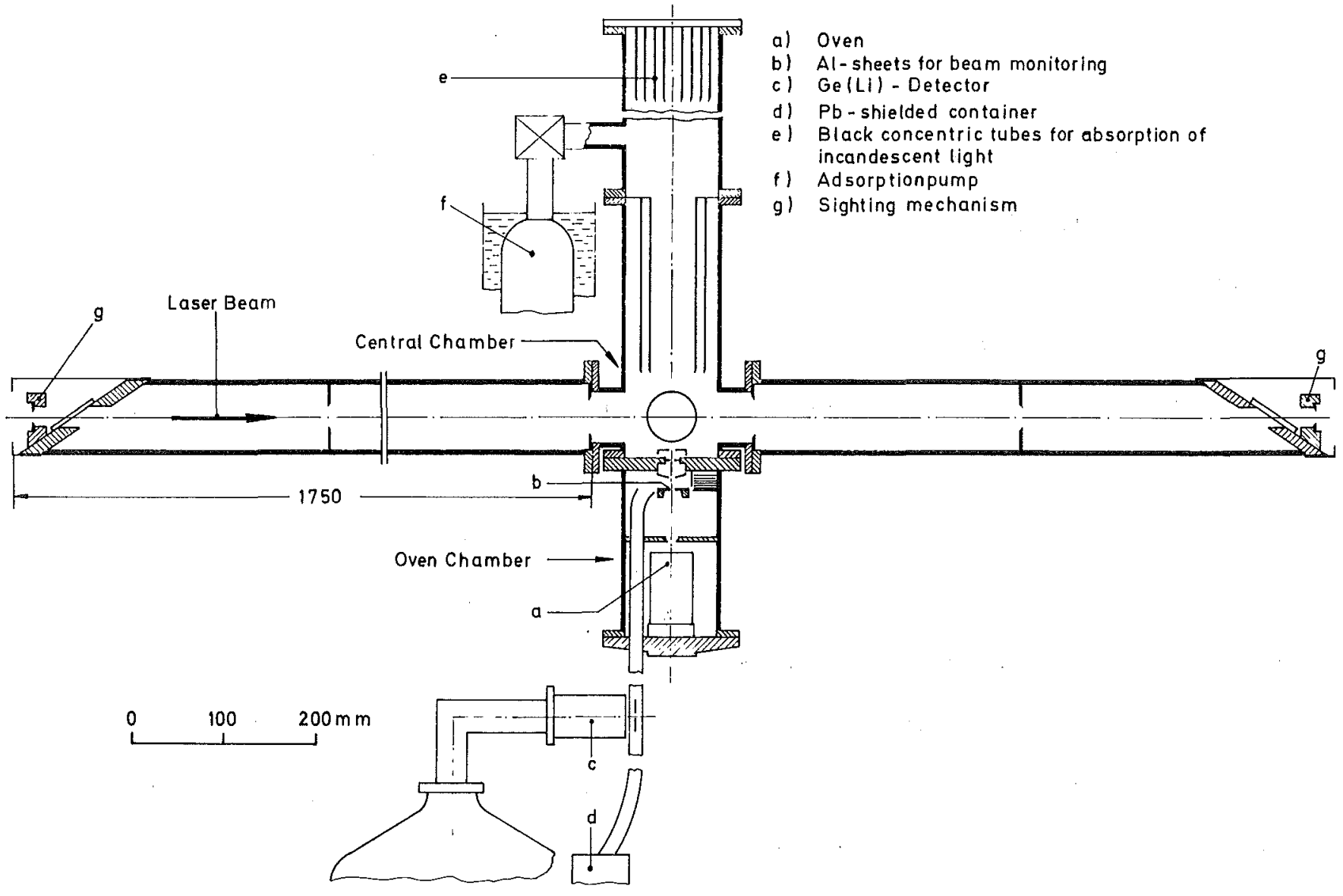

Fig. 3: Atomic beam apparatus cut in the plane of the laser beam and the atomic beam.

onto the entrance hole of a diaphragm in front of the photocathode and by large distances between this intersecting region and the entrance and exit windows of the laser beam, the main sources of scattered light. Thoroughly blackening of all inner surfaces was very helpful. Entrance and exit tubes of the laser beam have a relatively large diameter and are intersected by sharply edged anti-scattering diaphragms. A careful cleaning of the entrance and exit Brewster windows is important in order to end up with a remaining laser light background of 15 counts per sec and per mW laser input power. Background due to incandescent light of the heated oven has been reduced to 13 counts per sec at 


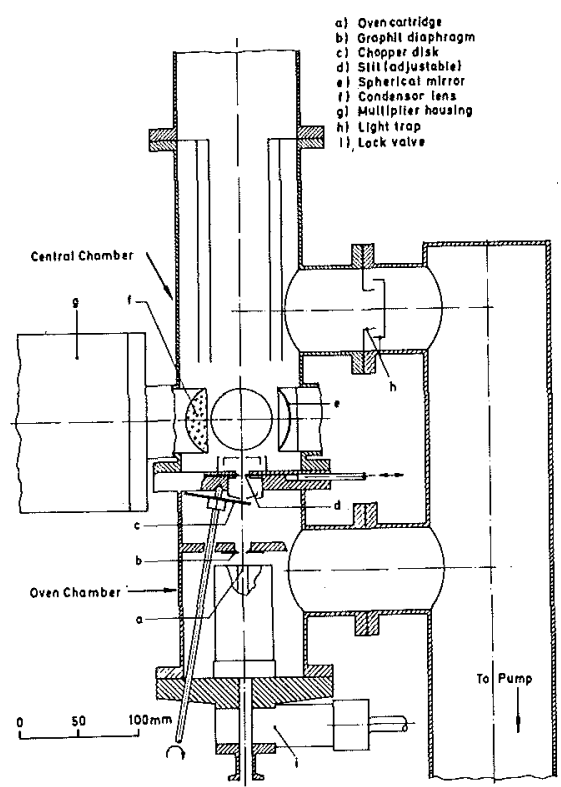

Fig. 4: Atomic beam apparatus cut in the plane perpendicular to the laser beam.

an oven temperature of $1600^{\circ} \mathrm{K}$. This is achieved by an infrared filter in front of the photomultiplier, by light traps inside the apparatus and, in particular, by a closed wall between the oven chamber and the part where the resonance transitions are induced. The collimating slit for the atomic beam is the only aperture in this wall. Finally, by cooling the photomultiplier cathode to about $260 \mathrm{~K}$ the background from the multiplier dark current is reduced to 50 counts per sec. In the cases of $128,131,133 \mathrm{~g}+\mathrm{m}_{\mathrm{Ba}}$ the background from $\gamma$-rays was actually negligible when the photomultiplier was shielded adequately. For higher $\gamma$-ray activities it may be necessary to improve the lead shielding of the multiplier. Thus, in measuring conditions the total background is less than 100 counts per sec so that a signal to background ratio of $2: 1$ is expected for $10^{4}$ resonance photons emitted per sec.

\subsection{Data acquisition}

A NOVA 2 computer interfaced to the experiment by a CAMAC system (s. fig. 5) controls the experiment and acquires the measured data. The difference frequency is set by the computer via a digital to analog converter. The photomultiplier pulses are fed into a fast preamplifier-discriminator (PAR 1120), 
the output pulses of which are counted by a $200 \mathrm{MHz}$ counter. This counter is gated by a current integrator measuring the time integral of the current from a photodiode monitoring the laser beam. In this way the photon counting result is normalized to the laser light intensity. The number of counts are displayed and stored together with the counting time. Setting the DAC to the next channel and thus tuning the laser frequency by a step of $1 \mathrm{MHz}$. the following counting cycle is started. Stable laser operation is checked visually by a scanning confocal Fabry-Perot showing single or multiple mode operation of the lasers, and by automatic monitoring of the frequency mixing signal. Cycles with unstable laser operation are rejected and repeated. As indicated above the atomic beam is controlled by $\gamma$-ray activity measurements, the results of which enable an off-line normalization to the actual atomic beam intensity.

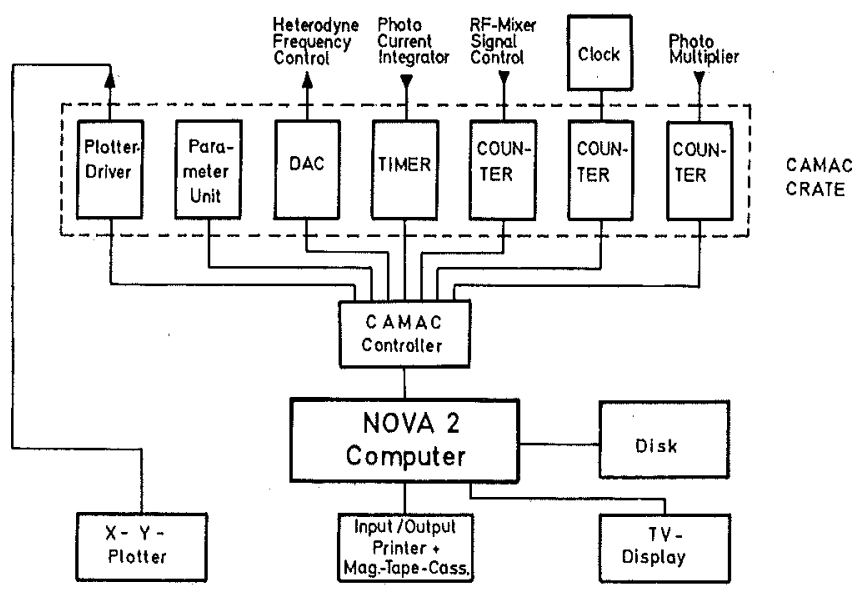

Fig. 5: Block diagram of the data acquisition system

\section{Sample preparation}

The neutron deficient instable Ba nuclides have been produced by nuclear reactions induced by charged particle or neutron irradiation of appropriate targets. Generally, a mixture of several isotopes has been produced, and due to long tails of the atomic resonance lines of neighboring isotopes, for an unambiguous assignment it is necessary to isolate the interesting isotope as well as possible. Moreover, in contrast to measurements of decay radiations 
the presence of stable isotopes is disturbing. Furthermore the barium samples have to be in elementary form which is not a trivial requirement in view of the chemical behaviour of $\mathrm{Ba}$. For these reasons an electromagnetic mass separater described elsewhere ${ }^{21}$ was used for sample preparation. With the mass separator the barium atoms are implanted into an adequate matrix material thus protected against oxidation. We used small silicon discs of ultra high purity as matrix material. Barium can be heated off from the silicon discs by moderate oven temperature to form an atomic beam source for a time duration of about some hours sufficient for a laser spectroscopic measurement. The efficiency of the mass separation ranges from 12 to $50 \%$ obtained by use of a surface-volume ionisation ion source with tantalum ionizer similar to ref. 22. After mass separation the total amount of barium in the beam oven is several $\mathrm{ng}$. Usually the oven material contains some p.p.m. of barium impurity. When heating the samples to $1500-1600 \mathrm{~K}$ this natural barium diffuses slowly out of the hot molybdenum and contaminates the atomic beam. Heating the open oven cells at $2100 \mathrm{~K}$ in high vacuum for several days before loading reduces the contamination by one order of magnitude. In the following some more details concerning the Ba isotopes measured (see fig. 6) are given.

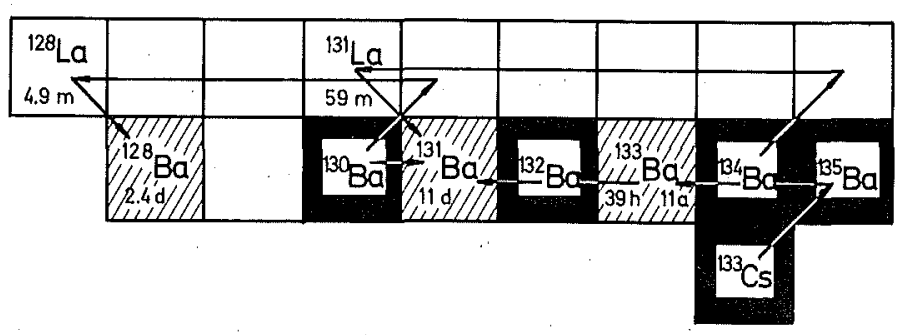

Fig. 6: Part of the chart of nuclides indicating the production of instable isotopes. 
a. 128 Ba

${ }^{128} \mathrm{Ba}\left(\mathrm{T}_{1 / 2}=2.4\right.$ d) has been produced by irradiating ${ }^{130} \mathrm{Ba}$ (enriched to $59 \%$ ) with $52 \mathrm{MeV}$ deuterons (beam current: $8.5 \mu \mathrm{A}$ ) from the Karlsruhe Isochronous Cyclotron for two days. ${ }^{128}$ La is produced via the ${ }^{130} \mathrm{Ba}(\mathrm{d}, 4 \mathrm{n})$-process and decays to ${ }^{128} \mathrm{Ba}$ with a half life of $5 \mathrm{~min}$. In order to obtain a ${ }^{128} \mathrm{Ba} / \mathrm{stable}$ Ba ratio as large as possible a relatively small amount of target material ( $\simeq 3 \mathrm{mg})$ has been irradiated with a high current density $\left(100 \mu \mathrm{A} / \mathrm{cm}^{2}\right)$ of the deuteron beam. The results displayed in fig. 9 have been obtained with a sample of about $6 \mathrm{ng}{ }^{128} \mathrm{Ba}$ as determined by $\gamma$-spectroscopy. The enrichment factor for the two nuclides ${ }^{128} \mathrm{Ba}$ and ${ }^{130} \mathrm{Ba}$ was in the order of $10^{4}$. $\underline{b} \underset{-}{131} \mathrm{Ba}$ In the case of ${ }^{131} \mathrm{Ba}\left(T_{1 / 2}=11.5 \mathrm{~d}\right)$ a much higher fraction of the target nuclei could converted into the radioactive isotope by irradiating isotopically enriched ${ }^{130}$ BaO with thermal neutrons at a flux $8 \cdot 10^{13} \mathrm{n} / \mathrm{cm}^{2} \mathrm{~s}$ for 7 days in Karlsruhe Research Reactor. This is due to a relatively large activation cross section $\left(\sigma_{n} \simeq 12.5 \mathrm{~b}\right)$ and longer irradiation time.. After mass separation a sample of $29 \mathrm{ng}{ }^{131}$ Ba was available.

C. $133 \mathrm{Ba}$

${ }^{133} \mathrm{Ba}$ has been produced via the reaction ${ }^{133} \mathrm{Cs}(\mathrm{d}, 2 \mathrm{n}){ }^{133} \mathrm{Ba}$ by irradiating

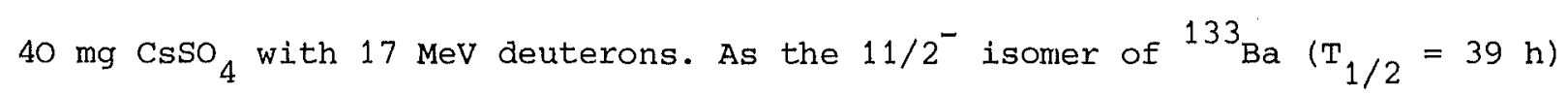
is produced simultaneously, the irradiated target was stored for some days before mass separation. Barium and cesium have been chemically separated by a carrier free ion exchange method 23 . For monitoring the subsequent mass separation $20 \mu \mathrm{g}$ of ${ }^{138} \mathrm{Ba}$ was added. The solution was then aried up before

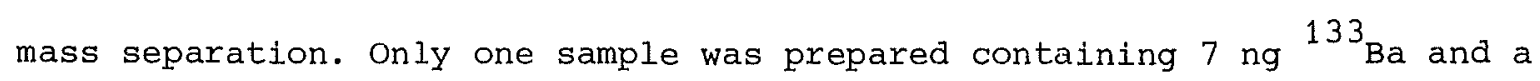
small amount $(<1 \mathrm{ng}){ }^{138} \mathrm{Ba}$. 
a. $133 \mathrm{~m}$

Increasing the deuteron energy to 26 - $30 \mathrm{MeV}$ favours the production of the $11 / 2^{-}$isomeric state of ${ }^{133} \mathrm{Ba}$, though the absolute yield is somewhat lower. $\mathrm{A} \mathrm{CsSO}_{4}$ target was irradiated for $20 \mathrm{~h}$ with a deuteron beam of $4.7 \mu \mathrm{A}$ and processed immediately after the end of irradiation. The final samples con-

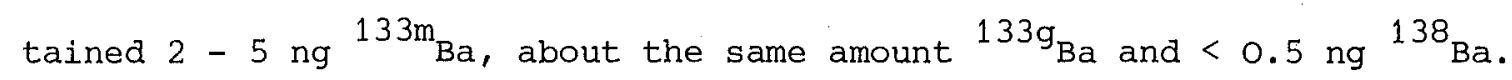

\section{Experimental results}

The very high resolution of our measurements is demonstrated in fig. 7 displaying a spectrum of a sample of mass separated stable isotopes ${ }^{137} \mathrm{Ba}$ and ${ }^{132} \mathrm{Ba}$ measured with reference to the transition in ${ }^{138} \mathrm{Ba}$. The line width of 21.5 $\mathrm{MHz}$ is only $10 \%$ larger than the natural line width so that the assumption of a pure Lorentzian is well justified for a leastsquare fit analysis of the spectra. In order to avoid power broadening and optical pumping effects the laser light intensity has been kept relatively low, $0.01-0.3 \mathrm{~mW}$. i.e. to an intensity density of less than $5 \mathrm{mw} / \mathrm{cm}^{2}$.

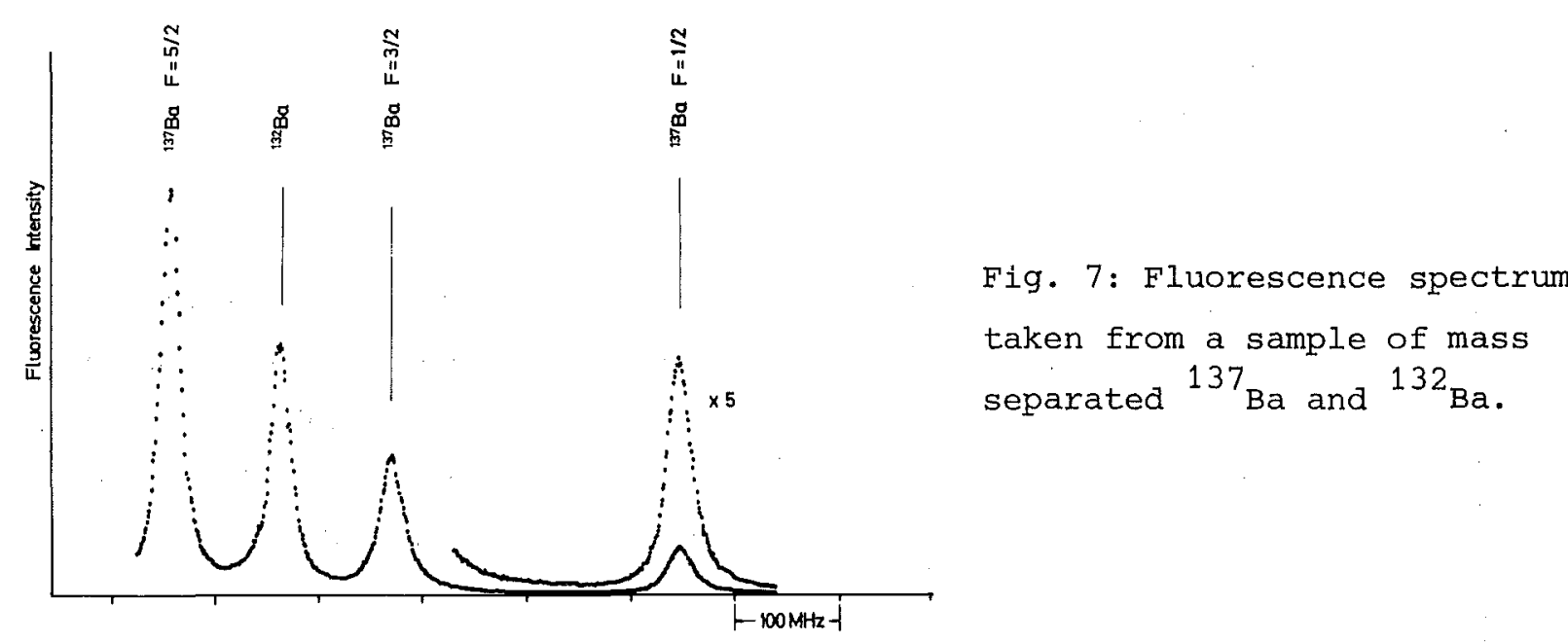

Higher intensities ( $\geq 1 \mathrm{~mW}$ ) have been used for inducing optical pumping with laser light of different polarizations which is a very helpful method for identyfing the different hyperfine structure components of the transitions in odd A isotopes. This is shown in fig. 8 for the $9 / 2$ transition of ${ }^{133 m} \mathrm{Ba}$. Exciting with 


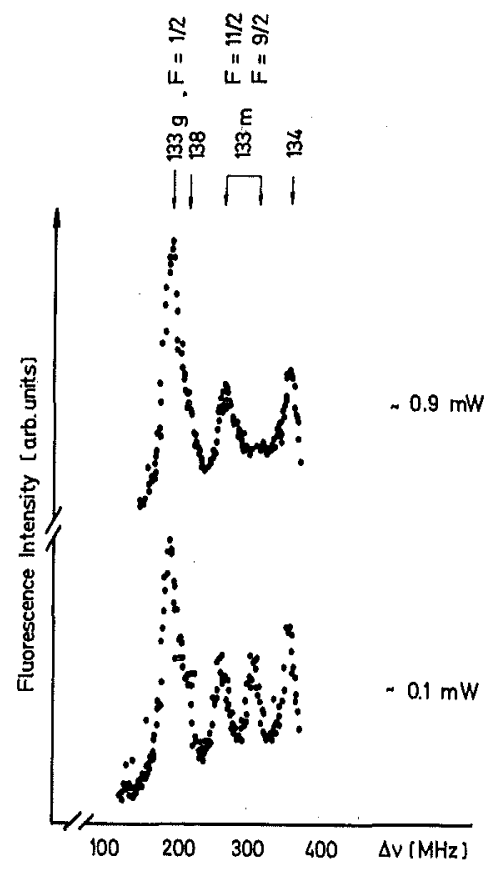

Fig. 8: Parts of two spectra from $133 \mathrm{~g}+\mathrm{m}_{\mathrm{Ba}}$ showing optical pumping of the $F=9 / 2$ hyperfine component when irradiating with linearly polarized laser light

increased power of linearily polarized $(\pi)$ laser light the $9 / 2$ component does not increase correspondingly. This effect is based on optical pumping into the $m_{F}= \pm 11 / 2$ magnetic substances of the groundstate from which excitation is forbidden due to the $\Delta \mathrm{m}=0$ selection rule. When pumping with circularily polarized $(\sigma \pm)$ light both the $9 / 2$ and $11 / 2$ components show such a behavior.

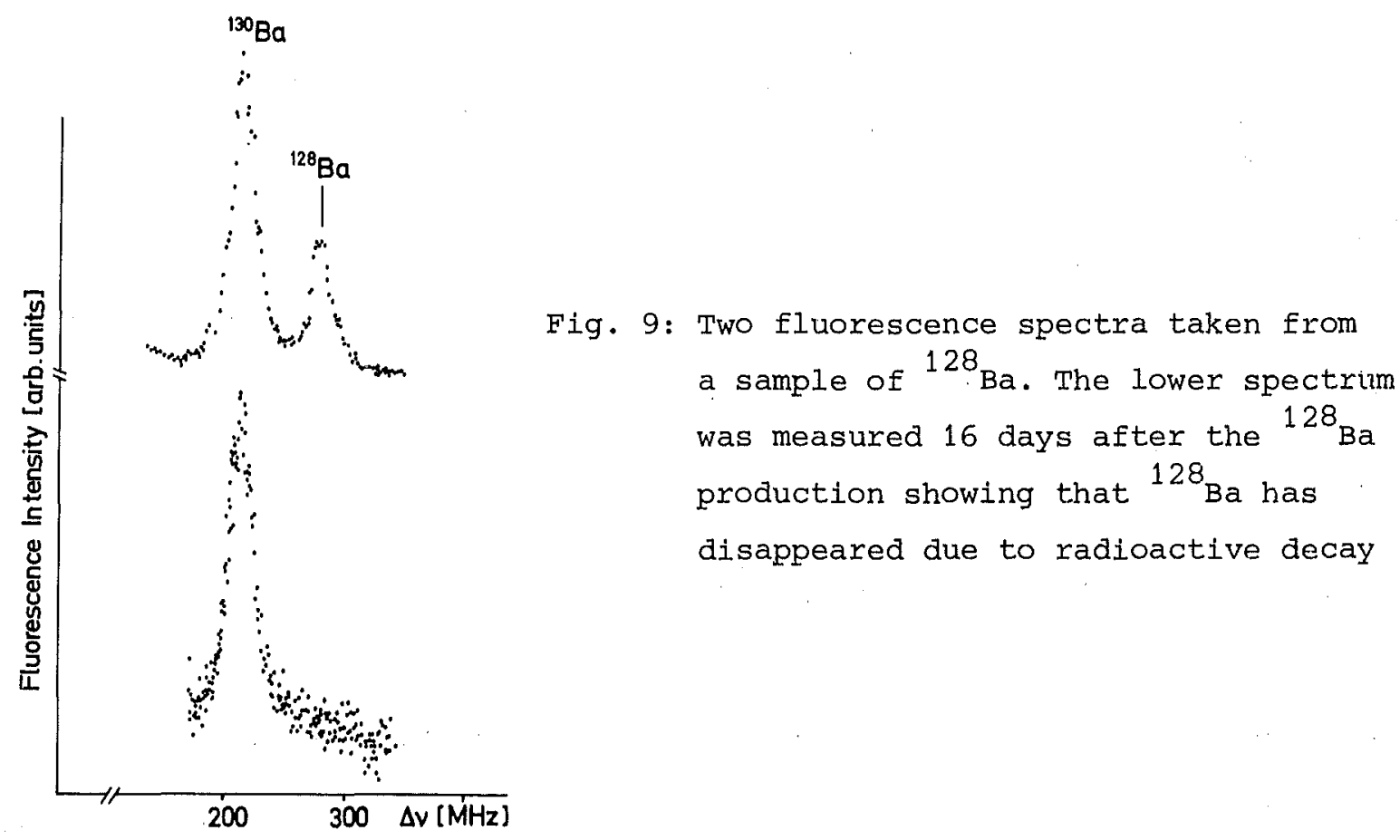


The radioactive decay of the nuclides and the corresponding decrease of the fluorescence light is a further means for the assigment of the resonance lines (for the case of ${ }^{128} \mathrm{Ba}$ see fig. 9).

A more complex spectrum and the result of the analysis is displayed in fig. 10

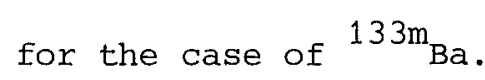

The counting rates in the resonance lines were typically $1-10 \mathrm{kHz}$, and the measuring time was about 1 - 5 s/channel.

The direct results for the frequency differences are compiled in tab. 1.

Tab. 2 gives the values for the isotope shifts, and of A- and B-factors of the hyperfine structure observed.

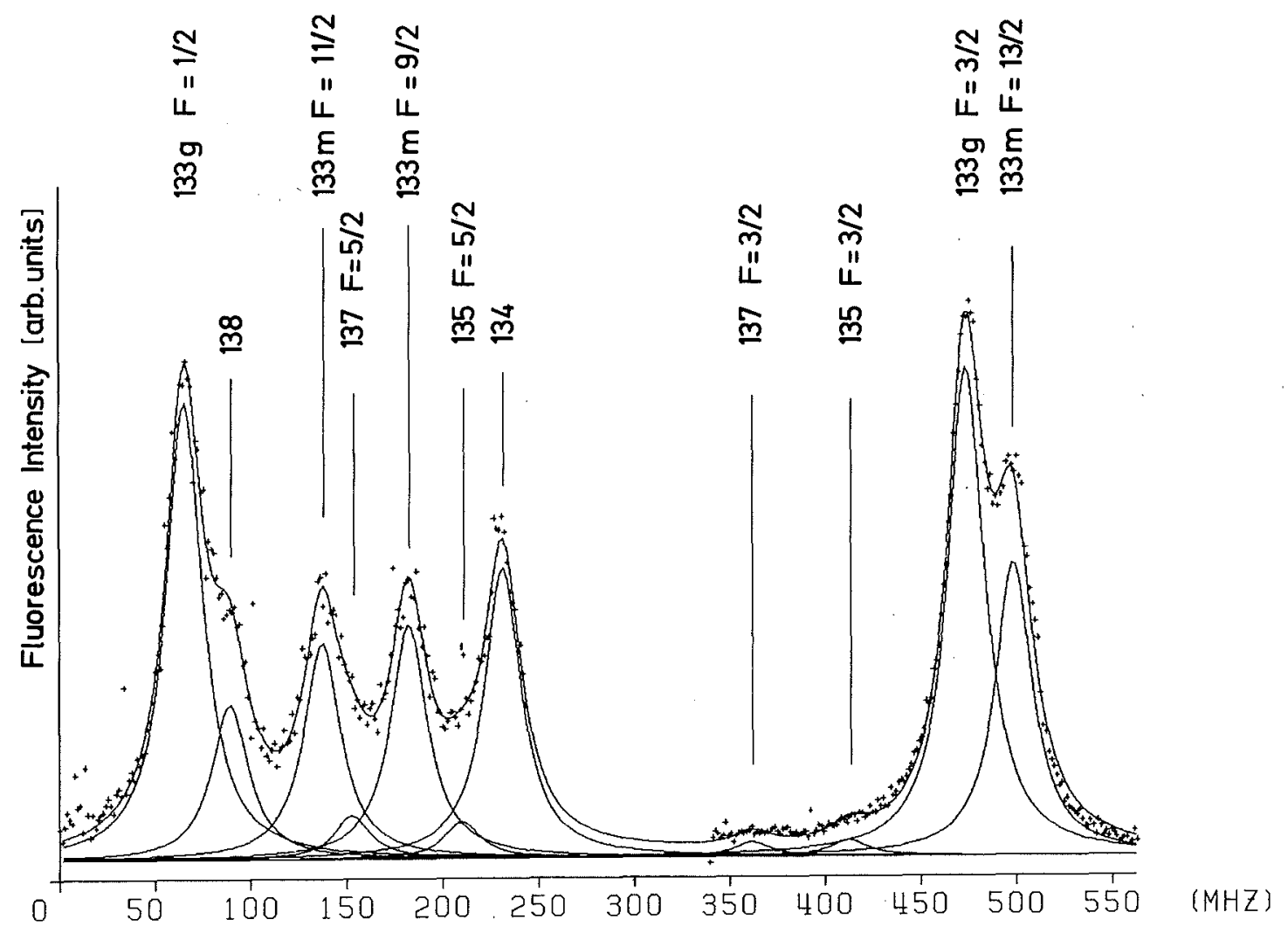

Fig. 10: Spectrum of a sample containing $133 \mathrm{~g}+\mathrm{m}_{\mathrm{Ba}}$. The solid curve represents the result of a leastsquares fit with purely Lorentzian shapes lines 
Tab. 1: $\mathrm{Ba} \mathrm{I}^{1} \mathrm{~S}_{\mathrm{O}}-{ }^{1} \mathrm{P}_{1}$ transition frequencies relative to the transition on ${ }^{138}$ Ba

\begin{tabular}{|c|c|c|c|c|}
\hline \multirow{2}{*}{$\frac{A}{140}$} & \multirow{2}{*}{$\frac{F}{1}$} & \multirow{2}{*}{$\frac{\Delta V[\mathrm{MHz}]}{-}$} & \multicolumn{2}{|c|}{ From other authors } \\
\hline & & & Marburg $^{a}:$ & -790.0 \\
\hline \multirow[t]{3}{*}{137} & $5 / 2$ & $.62 .8(6)$ & oxford ${ }^{b}$ : & $63.0(6)$ \\
\hline & $3 / 2$ & $275.1(6)$ & & $274.5(9)$ \\
\hline & $1 / 2$ & $551.5(6)$ & & $550.2(11)$ \\
\hline 136 & 1. & $128.9(5)$ & & $127.5(13)$ \\
\hline \multirow[t]{3}{*}{135} & $5 / 2$ & $121.6(6)$ & oxford ${ }^{b}$ : & $120.1(7)$ \\
\hline & $3 / 2$ & $326.6(6)$ & & $324.2(8)$ \\
\hline & $1 / 2$ & $547.2(6)$ & & $543.5(10)$ \\
\hline 134 & 1 & $143.0(5)$ & & $142.8(12)$ \\
\hline \multirow[t]{2}{*}{$133 \mathrm{~g}$} & $3 / 2$ & $386.5(9)$ & & \\
\hline & $1 / / 2$ & $-23.3(5)$ & & \\
\hline \multirow[t]{3}{*}{$133 m$} & $13 / 2$ & $411.3(9)$ & & \\
\hline & $11 / 2$ & $48.2(5)$ & & \\
\hline & $9 / 2$ & $93.8(5)$ & & \\
\hline 132 & 1 & $167.9(5)$ & & \\
\hline \multirow[t]{2}{*}{131} & $3 / 2$ & $373.8(10)$ & & \\
\hline & $1 / 2$ & $0.0(8)$ & & \\
\hline 130 & 1 & $207.3(7)$ & & \\
\hline 128 & 1 & $271.1(8)$ & & \\
\hline
\end{tabular}

a

from ref. 12

b from ref. 10 
Tab. 2: Isotope Shifts, A- and B-factors

\begin{tabular}{|c|c|c|c|c|c|c|}
\hline $\begin{array}{l}\text { Atomic } \\
\text { Number }\end{array}$ & $\begin{array}{c}\Delta v \\
{[\mathrm{MHz}]}\end{array}$ & $\begin{array}{c}\Delta \nu_{F} \\
{[\mathrm{MHz}]}\end{array}$ & $\begin{array}{c}\delta<r^{2}> \\
{\left[10^{-2} \mathrm{fm}^{2}\right]}\end{array}$ & $\mathrm{c}_{\mathrm{exp}} / \mathrm{C}_{\text {unif }}$ & $\begin{array}{c}\mathrm{A} \\
{[\mathrm{MHz}]}\end{array}$ & $\begin{array}{c}\mathrm{B} \\
{[\mathrm{MHz}]}\end{array}$ \\
\hline 140 & $-790.0^{a}$ & $-821^{a}$ & $23.2^{a}$ & & & \\
\hline 138 & 0. & & & & & \\
\hline 137 & $215.0(7)$ & $230(8)$ & $-4.9(2)$ & 0.44 & $-109.8(5)$ & $49.7(5)$ \\
\hline 136 & $128.9(5)$ & $160(16)$ & $-3.4(3)$ & 0.15 & & \\
\hline 135 & $260.9(7)$ & $308(24)$ & $-6.6(5)$ & 0.20 & $-98.3(5)$ & $32.5(4)$ \\
\hline 134 & $143.0(5)$ & $206(32)$ & $-4.4(7)$ & 0.10 & & \\
\hline $133 g$ & $250.0(9)$ & $332(41)$ & $-7.1(9)$ & 0.13 & $273.3(8)$ & \\
\hline $133 \mathrm{~m}$ & $202.0(10)$ & $284(4.1)$ & $-6.0(9)$ & 0.11 & $29.1(9)$ & $195.9(9)$ \\
\hline 132 & $167.9(5)$ & $267(50)$ & $-5.7(10)$ & 0.09 & & \\
\hline 131 & $249.2(21)$ & $363(57)$ & $-7.7(12)$ & 0.10 & $249.2(18)$ & \\
\hline 130 & $207.3(7)$ & $341(67)$ & $-7.2(14)$ & 0.08 & & \\
\hline 128 & $271.1(8)$ & $439(87)$ & $-9.3(18)$ & 0.08 & & \\
\hline
\end{tabular}

a) from reference 12 
5. Extraction of $\delta\left\langle r^{2}\right\rangle$ and nuclear moments

The isotope shift $\Delta v_{\text {IS }}$ of a spectral line of the transition frequency $v$ is usually split up into three contributions ${ }^{24}$ : the normal mass shift $\Delta \nu_{N M}$. the specific mass shift $\Delta \nu_{S M}$ and the field shift $\Delta \nu_{F}$. While the normal mass shift can be easily calculated by

$$
\Delta V_{N M}=\frac{m_{e}\left(M_{A^{\prime}}-M_{A}\right)}{M_{A} \cdot M_{A^{\prime}}} \nu \simeq \frac{m_{e}}{m_{N}} \quad \frac{A^{\prime}-A}{A \cdot A^{\prime}} \nu
$$

(with $m_{e^{\prime}} m_{N}$ the electron and nucleon mass, respectively, $M_{A}$, and $M_{A}$ the masses and atomic numbers of the two nuclides), the specific mass shift which is due to the correlations of the momenta of the atomic electrons $\left(<\sum_{i>j} \vec{p}_{i} \cdot \vec{p}_{j}>\right)$, is subject of various uncertainties. It may be calculated within a Hartree Fock procedure ${ }^{25}$, but in most cases a quantitative description on purely theoretical ground fails. Another method to rule out the specific mass effect is based on the comparison with sufficiently accurate isotope shift measurements of electronic $\mathrm{K}_{\alpha}-\mathrm{x}$-rays ${ }^{8}$ or of muonic $\mathrm{x}-\mathrm{rays}{ }^{7}$. Such a comparison (King-Plot ${ }^{25}$ ) results in $\Delta \nu_{\mathrm{SM}}=(0 \pm 1) \Delta \nu_{\mathrm{NM}}$ for the case of $\mathrm{Ba}$. This is in agreement with arguments for small values of $\Delta v_{S M}$ in $(s-p)$ transitions ${ }^{26}$. It should be noted, however, that the choice of $\Delta v_{S M}=0$ is somewhat arbitrary and is actually a source of considerable uncertainty in the analysis of the direct experimental results. A smaller uncertainty in $\Delta V_{S M}$ would require extremely precise information on isotope shifts of electronic $\mathrm{Ba} \mathrm{K}_{\alpha}$-X-rays which is beyond experimental reach. More precise muonic Ba $\mathrm{X}$-ray data would improve the accuracy of the King plot even if strictly speaking the comparison of optical and muonic X-ray shifts includes some additional problems. The remaining field shift $\Delta \nu_{F}$, given in tab. 2 with errors mainly arising from the uncertainties in $\Delta \nu_{S M}$ originates from the influence of the nuclear charge distributions and is connected with the change of the radial moments 27 


$$
\Delta \nu_{F}=F\left\{\delta<r^{2}>+\left(C_{2} / C_{1}\right) \delta\left\langle r^{4}>+\ldots\right\}\right.
$$

Neglecting higher moment terms and using the electronic factor $F$ given by Fischer et al. ${ }^{12}$ values of $\delta\left\langle r^{2}\right\rangle$ shown in fig. 11 are obtained. In tab. 2 the ratios $\mathrm{C}_{\text {exp }} / \mathrm{C}_{\text {unif }}$ of the Is constants (with $\mathrm{C}_{\text {unif }}$ based on a uniformly charged sphere of the radius $R=1.2 \mathrm{~A}^{1 / 3} \mathrm{fm}$ ) are also given.

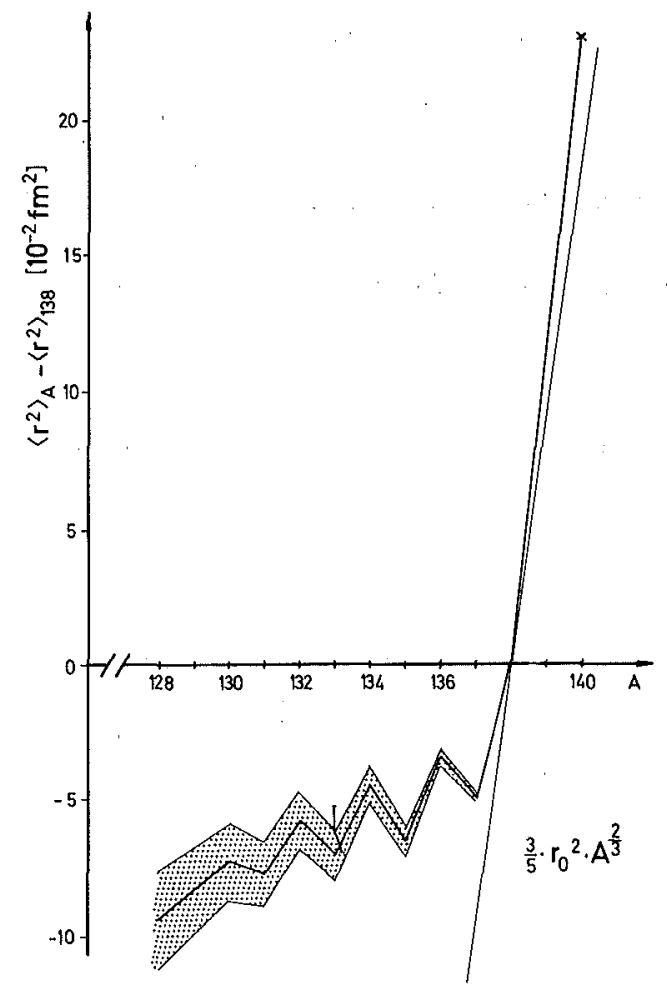

Fig. 11: Differences of rms charge radii of Barium isotopes. The straight line represents the $\mathrm{A}$ dependence expected for the standard homogenous sphere with $R_{0}=r_{0} A^{1 / 3}$.

Magnetic dipole and electric quadrupole moments of odd Ba isotopes have been obtained from hyperfine structure studies of the $6 \mathrm{~s} 6 \mathrm{p}{ }^{1} \mathrm{P}_{1}$ states by several authors, with high accuracy in particular for ${ }^{137} \mathrm{Ba}$ and ${ }^{135} \mathrm{Ba}$ (see refs. 14,15 and 40 and references given there). Neglecting the hyperfine anomaly the magnetic moments $\mu_{I}$ of $131,133 \mathrm{~g}, 133 \mathrm{~m}_{\mathrm{Ba}}$ are derived from the magnetic dipole constants $A$ (see tab. 2) and the known values of $A\left({ }^{135_{B a}}\right.$ ) and of $\mu_{I}\left({ }^{135_{B a}}\right)$. Höhle et al. ${ }^{13}$ estimated a hyperfine anomaly between ${ }^{135} \mathrm{Ba}$ and ${ }^{13} \mathrm{Ba}$ of about $0.5 \%$ from the hyperfine structure measurement of the Ba II $\left(6 \mathrm{~s}^{2} \mathrm{~s}_{1 / 2}-\right.$ $6 \mathrm{p}^{2} \mathrm{P}_{1 / 2(3 / 2)}$ ) transition. Similarily, we refer to the known results 14 for $135,137 \mathrm{Ba}$ when extracting a Q-value from our value of $\mathrm{B}\left({ }^{133 \mathrm{~m}_{\mathrm{Ba}}}\right)$ and avoid 
a more detailed discussion necessary in the theoretical interpretation of B-values ${ }^{28}$. Sternheimer ${ }^{29}$ correction is not included as it has not been calculated for the configurations considered here.

\section{Discussion}

Comparing the extracted $\delta\left\langle r^{2}>\right.$ values to values from a uniformly charged sphere with $R=1.2 \mathrm{~A}^{1 / 3} \mathrm{fm}$ (see fig. 1 ), it is evident that the rms charge radii of the neutron deficient Ba nuclei decrease much more slowly with decreasing neutron number than expected from the simple $A^{1 / 3}$ rule*. This feature may be attributed to an increasing mean square ground state deformation $\left\langle\beta^{2}\right\rangle$ of these transitional nuclei. The deformation effect in the values of $\delta\left\langle r^{2}\right\rangle$ can be described by

$$
\delta\left\langle r^{2}\right\rangle=\delta\left\langle r^{2}\right\rangle_{0}+\frac{5}{4 \pi}\left\langle r^{2}\right\rangle_{0} \delta\left\langle\beta^{2}>\right.
$$

where $\delta\left\langle r^{2}\right\rangle_{0}$ is the variation of a purely spherical charge distribution. Assuming explicitely that $\delta\left\langle r^{2}\right\rangle_{0}$ is given by the $A^{1 / 3}$ law with a common radius parameter $r_{O}$ (equivalent radius $R_{e q}=\sqrt{\frac{5}{3}} \cdot\left\langle r^{2}\right\rangle_{O}^{1 / 2}=r_{O} \cdot A^{1 / 3}$ ) and fixing the value $r_{0}(\simeq 1.2)$ via the equation

$$
\left\langle r^{2}\right\rangle=\left\langle r^{2}\right\rangle_{O}\left(1+\frac{5}{4 \pi} B_{O}^{2}\right)
$$

with the electron scattering result $\left\langle\mathrm{r}^{2}\right\rangle^{1 / 2}=3.836 \mathrm{fm}$ for ${ }^{138} \mathrm{Ba}$ and the value $\beta_{O}=0.090$ from the $B\left(E 2, O^{+} \rightarrow 2^{+}\right)$value ${ }^{30}$, this procedure results in $\left\langle B^{2}\right\rangle^{1 / 2}=$ $\left.\left(\delta<\beta^{2}\right\rangle+\left\langle\beta^{2}\right\rangle^{138}\right)^{1 / 2}$ and $\delta<\beta^{2}>$ values listed in tab. 3. Corresponding rms deformation values derived from measured ${ }^{30} \mathrm{~B}\left(\mathrm{E} 2, \mathrm{O}^{+} \rightarrow 2^{+}\right)$values $\left(\beta_{\text {trans }}\right)$ or from quadrupole moments $\left(B_{Q}\right)$ of the first excited $2^{+}$states 30,31 (implying the rotational model) prove to be significantly smaller. This reflects the so

The actual slope is somewhat affected by the uncertainties in the specific mass shift. 
called isotope shift discrepancy ${ }^{32}$ observed as an overall effect in all mass regions and often taken into account by empirical factors of $\delta\left\langle r^{2}\right\rangle_{0}$ in eq. 6.1 . Certainly the assumption of a monotonic behavior of $\delta\left\langle r^{2}\right\rangle_{0}$ is not well justified, and the charge distribution may contract less when neutrons are stripped as would be expected naively. Therefore we have to conclude that a large part of the difference between measured $\delta<x^{2}>$ values and simple predictions according to the $\mathrm{A}^{1 / 3}$ rile is connected with such an effect which may provide some information on the nuclear compressibility 17 . We may account for the (negative) compression by introducing a term $\delta<\tilde{r}^{2}>$ into eq. 6.1 and writing

$$
\delta\left\langle r^{2}\right\rangle=\delta\left\langle r^{2}\right\rangle_{0}+\delta\left\langle\tilde{r}^{2}>+\frac{5}{4 \pi}\left\langle r^{2}\right\rangle_{O} \delta<B^{2}>\right.
$$

Using as input numbers the variations in $\beta_{\text {trans }}$ the $\delta<\tilde{r}^{2}>$ values given in tab. 3 represent the contribution to the observed values $\delta\left\langle r^{2}\right\rangle$.

Tab. 3: Staggering parameters $\gamma_{\text {stag }}$ deformations and compression $\delta<\tilde{r}^{2}>$

\begin{tabular}{|c|c|c|c|c|c|}
\hline $\mathrm{A}$ & $\gamma_{\text {stag }}$ & $\begin{array}{l}\delta<\beta^{2}> \\
{\left[1 a^{-2}\right]}\end{array}$ & $<\beta^{2}>1 / 2$ & $B_{\text {trans }}^{a}$ & $\begin{array}{l}\delta<\tilde{r}^{2}> \\
{\left[10^{-2} \mathrm{fm}^{2}\right]}\end{array}$ \\
\hline 138 & & & 0.090 & $0.090(2)$ & 0 \\
\hline 137 & -0.88 & $0.69(2)$ & 0.123 & & \\
\hline 136 & & $2.09(4)$ & 0.170 & $0.125(2)$ & +12.3 \\
\hline 135 & -4.40 & $2.99(6)$ & 0.195 & & \\
\hline 134 & & $4.49(7)$ & 0.230 & $0.160(2)$ & +25.0 \\
\hline $133 g$ & -2.15 & $5.48(10)$ & 0.251 & & \\
\hline $133 \mathrm{~m}$ & -0.46 & $5.60(10)$ & 0.253 & & \\
\hline 132 & & $6.93(12)$ & 0.278 & & \\
\hline 131 & -0.67 & $8.02(13)$ & 0.297 & & \\
\hline 130 & & $9.40(16)$ & 0.320 & $0.23(1)$ & +44.0 \\
\hline 128 & & $11.87(20)$ & 0.356 & $0.26 \quad(3)$ & +52.4 \\
\hline
\end{tabular}

a) The values of $B_{\text {trans }}$ are calculated from $\mathrm{B}\left(\mathrm{E} 2, \mathrm{O}^{+} \rightarrow 2_{1}^{+}\right)$-values given in ref. 30 , in the case of ${ }^{128} \mathrm{Ba}$ from ref. 39. 
A more detailed feature seen in fig. 11 is the prominent odd-even staggering of the $\delta\left\langle r^{2}\right\rangle$ values. Obviously the odd isotopes are smaller than the neighboring even isotopes which results in large negative values (see tab. 3) of the staggering parameter (with A even)

$$
\gamma_{\text {stag }}=\frac{\left\langle\mathrm{r}^{2}\right\rangle_{A+1}-\left\langle\mathrm{r}^{2}\right\rangle_{A}}{\frac{1}{2}\left(\left\langle\mathrm{r}^{2}\right\rangle_{A+2}-\left\langle\mathrm{r}^{2}\right\rangle_{A}\right)}
$$

These values differ considerably even in sign from theoretical predictions for ${ }^{137} \mathrm{Ba}$ and ${ }^{135} \mathrm{Ba}$ calculated by Reehal and Sorensen ${ }^{33}$ on the basis of the pairing-plus-quadrupole model including the blocking of the groundstate correlation by the unpaired quasiparticle (Note that the experimental errors in $\delta\left\langle r^{2}\right\rangle$ due to the uncertainty of the specific mass shift contribution do not affect the experimental $\gamma_{\text {stag }}$-values).

Tab. 4: Spectroscopic (Q), intrinsic $\left(Q_{0}\right)$ quadrupolmoments, the corresponding deformation $B_{Q}$ and magnetic moments $\mu_{I}$

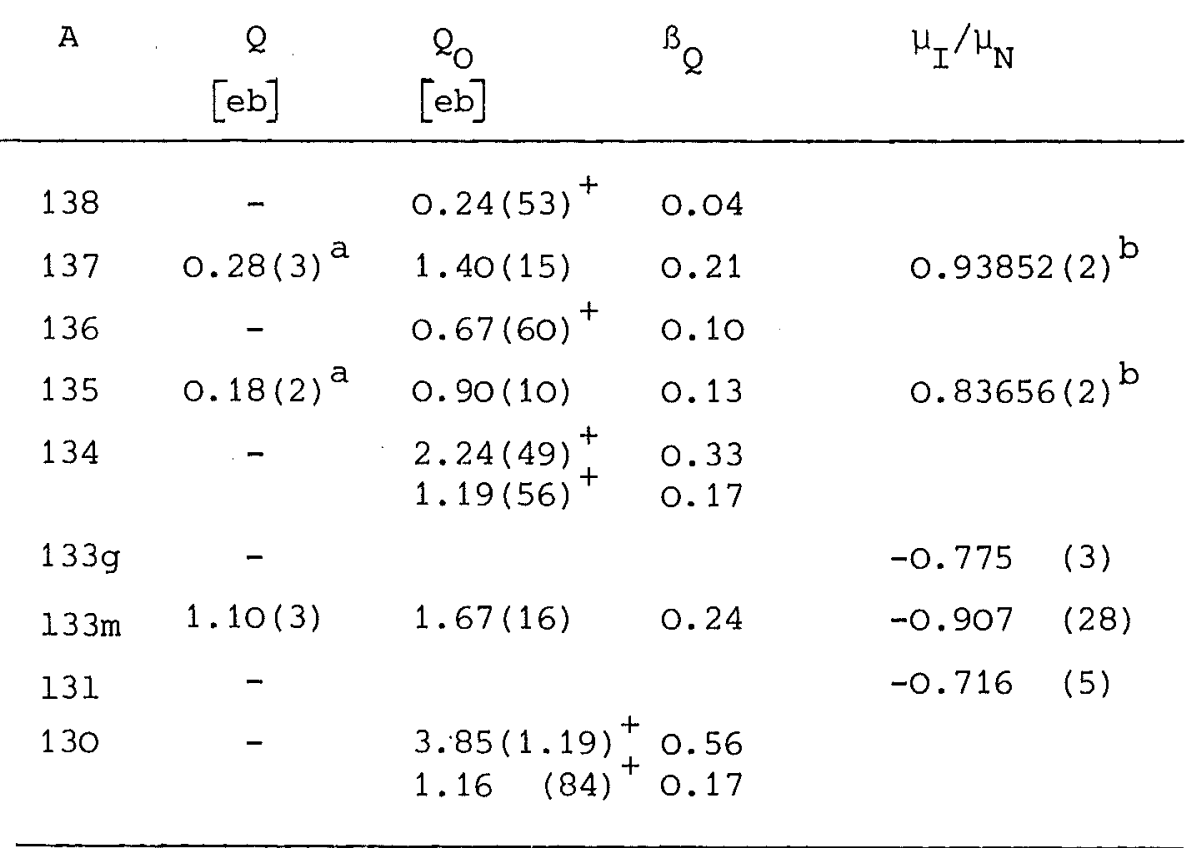
a) from ref. 14
b) from ref. 40

+) derived from $Q\left(2_{1}^{+}\right)$on the basis of the rotational model (see ref. $39,31,41$ ) 
The spectroscopic quadrupole moments $Q$ of $137,135,133 \mathrm{~m}_{\mathrm{Ba}}$ may be compared with values of the deformation parameters deduced from the discussion of the field shift (tab. 4). Such a comparison is strongly affected by the choice of the particular model connecting these quantities. Using the projection formula of the strong coupling scheme

$$
Q_{0}=\frac{(I+1) \cdot(2 I+3)}{I(2 I-1)} Q
$$

for a $K=I$ state intrinsic quadrupole moments $Q_{O}$ are deduced which are in the order of magnitude of the $Q_{0}$ values for the $2^{+}$states of the even Ba-isotopes 30,31 . The corresponding values of the deformation parameter $B_{Q}$ are also given in tab. 4. Strictly, the three quantities $\left\langle\beta^{2}\right\rangle^{1 / 2}, \beta_{\text {trans }}$ and $\beta_{Q}$ are of different type and are only related by a nuclear model specifying radial and angular shape of the nucleus. In the case of a perfect rotator the three values should be identical, while the vibrational model requires $\left\langle\beta^{2}\right\rangle^{1 / 2}=\beta_{\text {trans }}$ and $\beta_{Q}=0$. Transitional nuclei have to be described more adequately by a generalized model ${ }^{34-37}$ with a more complex relation of the three quantities.

The observed values of the magnetic moments of ${ }^{135} \mathrm{Ba}$ and ${ }^{137} \mathrm{Ba}$ are in fair agreement with the theoretical predictions of Kisslinger and Sorensen 38 whereas those of $131,133 g_{B a}$ are larger by a factor 2 than the theoretical values.

We thank Prof. Dr. H. Walther for his encouraging interest and advice when starting our experimental investigation, and Prof. Dr. E.W. Otten and Dr. D.N. Stacey for stimulating discussions. The technical assistance of Mr. B. Feurer in preparing the mass separated samples is gratefully acknowledged. 


\section{$\underline{\text { References }}$}

1. E. Arimondo, M. Inguscio and P. Violino, Rev. Mod. Phys. 49 (1977) 31

2. W. Demtröder, Physics Report - Phys. I. 7C (1973) 225

3. J. Bonn, G. Huber, H.J. Kluge and E.W. Otten, Z. Phys. A276 (1976) 203

4. J. van Klinken, D. Habs, H. Klewe-Nebenius, K. Wisshak, G. Nowicki,

J. Buschmann, S. Göring, R. Löhken, H. Rebel and G. Schatz, KFK-Report 1978 (1974)

5. D. Habs, H. Klewe-Nebenius, K. Wisshak, R. Löhken, G. Nowicki, H. Rebel, z. Physik 267 (1974) 149

6. J. Heisenberg, R. Hofstadter, J.S. McCarthy, I. Sick, M.R. Yearian, B.C. Clark, R. Herman and D.G. Ravenhall in Topics of Modern Physics: Tribute to E.U. Condon, ed. by W.E. Brittin and H. Odabasi (Adam Hilger, London) p. 169

7. R. Engfer, H. Schneuwly, J.L. Vuilleumier, H.K. Walter and A. Zehnder, Atomic Data and Nuclear Data Tables 14 (1974) 613

8. O.T. Sumbaev, A.F. Mezentsev, V.I. Marushenko, A.S. Ryl'nikov and G.A. Iwanov, Sov. Journ. of Nucl. Phys. 9 (1969) 529

9. D.A. Jackson and H.T. Duong, Proc. Roy. Soc. A280 (1964) 323 See also: K. Heilig, Spectrochimica Acta $32 \mathrm{~B}$ (1977) 1

10. P.E.G. Baird, R.J. Brambley, K. Burnett, D.N. Stacey, D.M. Warrington, G.K. Woodgate, to be published (private communication)

11. H.J. Kluge and S. Sauter, Z. Phys. 270 (1974) 295

12. W. Fischer, M. Hartmann, H. Hühnermann and H. Vogg, Z. Phys. 267 (1974) 209

13. C. Höhle. H. Hühnermann, Th. Meier, H.R. Ihle and R. Wagner, Phys. Lett. $\underline{62 \mathrm{~B}}$ (1976) 390

14. I.J. Ma and G. zu Putzlitz, Z. Phys. A277 (1976) 107

15. M.W. Swagel and A. Lurio, Phys. Rev. 169 (1969) 114

L. Olschewski and E.W. Otten, Z. Phys. 196 (1966) 77 
16. G. Nowicki, K. Bekk, S. Göring, H. Hanser, H. Rebel and G. Schatz, Phys. Lett. 39 (1977) 332

17. G.E. Brown, in Facits of Physics, ed. by A. Bromley and V.W. Huhes (Academic Press, New York, 1970) p. 141

18. H.W. Kogelnik, E.I. Ippen, A. Dienes, C.V. Shank, IEEE Journ. Quant. Electr. $\underline{\mathrm{QE}-8}(1972) 373$

19. W. Hartig and H. Walther, Appl. Phys. 1 (1973) 171, H.Hartwig, Diplomarbeit, Cologne University, 1973 (unpublished)

20. G.W. Greenless, D.L. Clark, S.L. Kaufmann, D.A. Lewis, I.F. Tonn and I.H. Broadhurst, Opt. Comm. 23 (1977) 236

21. H. Fabricius, K. Freitag and S. Göring, Nucl. Instr. Meth. 38 (1965) 64 H. Fabricius, K. Freitag, S. Göring, A. Hanser and H.-J. Langmann, KFK-Report 511 (1966)

22. C. Beyer, E. Hermann, A. Piotrowski, V.I. Raiko and H. Tyroff, Nucl. Instr. Meth. 96 (1971) 437

23. R. Shabana and H. Ruf, KFK-Report 2460 (1977)

24. H. Kopfermann, Nuclear Moments - Academic Press New York, 1958, D.N. Stacey, Rep. Progr. Phys. 29 (1966) 171

25. J. Bauche and R.J. Champeau, Advances in Atomic and Molecular Physics, Vol. 12 (1976), ed. D.R. Bates and Benjamin Bederson, Academic Press, New York, San Francisco - London

26. K. Heilig and A. Steudel; Atomic Data and Nuclear Data Tables 14 (1974) 613

27. E.C. Seltzer, Phys. Rev. 188 (1969) 1916

28. G. Breit and L.A. Wills, Phys. Rev. 44 (1933) 410

29. R.M. Sternheimer, Phys. Rev. 80 (1950) 102, 164 (1967) 10

30. A. Christy and 0 . Häusser, Nuclear Data Tables 11 (1972) 281

31. A.M. Kleinfeld, A. Bokisch and K.P. Lieb, Nucl. Phys. A283 (1977) 526

32. E.W: Otten, Hyperfine Interactions $\underline{2}$ (1976) 127 
33. B.S. Reehal and R.A. Sorensen, Nucl. Phys. A161 (1971) 385

34. G. Gneuß and w. Greiner, Nucl. Phys. A171 (1971) 449

35. J. Meyer-ter-Vehn, Nucl. Phys. A249 (1975) 111, (1975) 141

36. H. Toki and A. Faessler, Nucl. Phys. A253 (1975) 231

37. J. Dobaczewski, S.G. Rohoziński and J. Srelny, Z. Physik A282 (1977) 203 S.G. Rohoziński, J. Dobaczewski, B. Nerlo-Pomorska, K. Pomorski and J. Srelny, Nucl. Phys. A292 (1977) 66

38. L.S. Kisslinger and R.A. Sorensen, Rev. Mod. Phys. 35 (1963) 853

39. W. Kutschera, W. Dehnhardt, O.C. Kistner, P. Kump, B. Povh and H.J. Sann, Phys. Rev. C5 (1972) 1658

40. L. Olschewski, Z. Physik 249 (1972) 205

41. M. Neimann, R. Kalish, D.R. Somayajulu, B. Herskind, F. Genovese and L. Grodzins, Phys. Lett. $\underline{52}$ (1974) 189 
Appendix: Identification of the hyperfine structure components by optical pumping processes.

In the BaI $6 \mathrm{~s}^{2}{ }^{1} \mathrm{~s}_{\mathrm{o}}-6 \mathrm{~s} 6 \mathrm{p}{ }^{1} \mathrm{P}_{1}$ transition the hyperfine structure is due to the splitting of ${ }^{1}{ }_{P_{1}}$ state into two components $F=3 / 2$, $1 / 2$ for a $\mathrm{Ba}$ nucleus with the nuclear spin $I=1 / 2$ or into three components $F=I+1, I, I-1$ for $I>1 / 2$. In order to be more specific we discuss the technique of identifying the different hyperfine components by optical pumping processes in the case of $I=3 / 2$. In figs. $\mathrm{A} 1$ and $\mathrm{A} 2$ the transitions between the (2F+1) magnetic substates $m_{F}=-F,-F+1 \ldots F$ of the ${ }^{1} S_{\circ}$ and ${ }^{1}{ }_{P}$ level (with $F=5 / 2,3 / 2$ and $1 / 2$, respectively) are schematically indicated (Kastler diagrams). These electric dipole transitions have the selection rules

$$
\begin{array}{ll}
\Delta \mathrm{F}=0, \pm 1 & 0 \leftrightarrow 0 \text { forbidden } \\
\Delta \mathrm{m}_{\mathrm{F}}=0, \pm 1 &
\end{array}
$$

In transitions with $\Delta \mathrm{m}_{\mathrm{F}}=\mathrm{O}$ ( $\pi$-transitions) the light is linearly polarized ( $E$ vector parallel to the external magnetic field applied when observing the Zeeman effect), $\sigma_{+}$-light $\left(\Delta \mathrm{m}_{\mathrm{F}}= \pm 1\right)$ is circularily polarized. Excitating the Ba atoms with linearly polarized light the hyperfine component $F=1 / 2$ of the ${ }^{1} P_{1}$ level e.g. can be populated only from the $\mathrm{m}_{\mathrm{F}}= \pm 1 / 2$ sublevels of ${ }^{1} \mathrm{~S}_{\mathrm{O}}$ (quantization axis taken in the direction of E-vector). But deexcitation occurs also with $\Delta m_{F}= \pm 1$ into the $m_{F}= \pm 3 / 2-{ }^{1} S_{0}$ substates from where this hyperfine component cannot be reexcited.

Consequently, if the light intensity is high enough so that multiple excitation is occurring the atoms will be pumped into the $m_{F}= \pm 3 / 2$ level and the fluorescence signal will go into saturation. In the case of the $F=3 / 2 \rightarrow 3 / 2$ and $F=3 / 2 \rightarrow 5 / 2$ hyperfine components such a pumping effect with $\pi$ light is not possible. The fluorescence light increases linearly with the laser light intensity. Generally, applying $\pi$-light excitation to nuclei with $I>1 / 2$ only the hyperfine component $F=I-1$ shows saturation effects.

Exciting the ${ }^{1}{ }_{P_{1}}$ hyperfine components with circularily polarized light in addition to the $F=I-1$ component also the $F=I$ component can be affected by optical pumping (for $I>1 / 2$ ) as indicated for $\sigma^{+}$light $\left(\Delta \mathrm{m}_{\mathrm{F}}=+1\right)$ in fig. A2. For $\mathrm{I}=1 / 2$ optical pumping can be induced for the $F=1 / 2$ component with $\sigma$-light. 
It should be noted that in addition to pumping effects the angular distribution of the fluorescence radiation following the excitation with polarized light provides some information easily observed by changing the polarization direction.
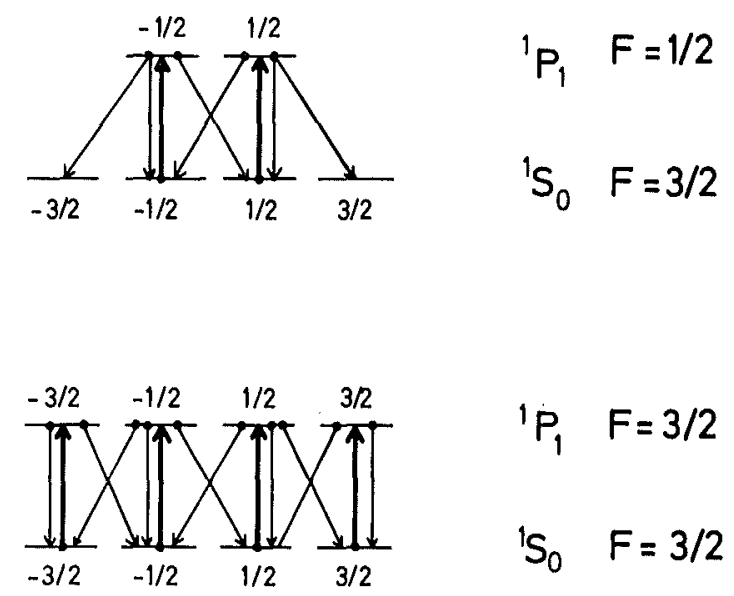

Fig. A1:

Optical pumping effects with $\pi$-light in a ${ }^{1} \mathrm{~S}_{\mathrm{O}}-{ }^{1} \mathrm{P}_{1}$ transition for a $I=3 / 2$ nucleus
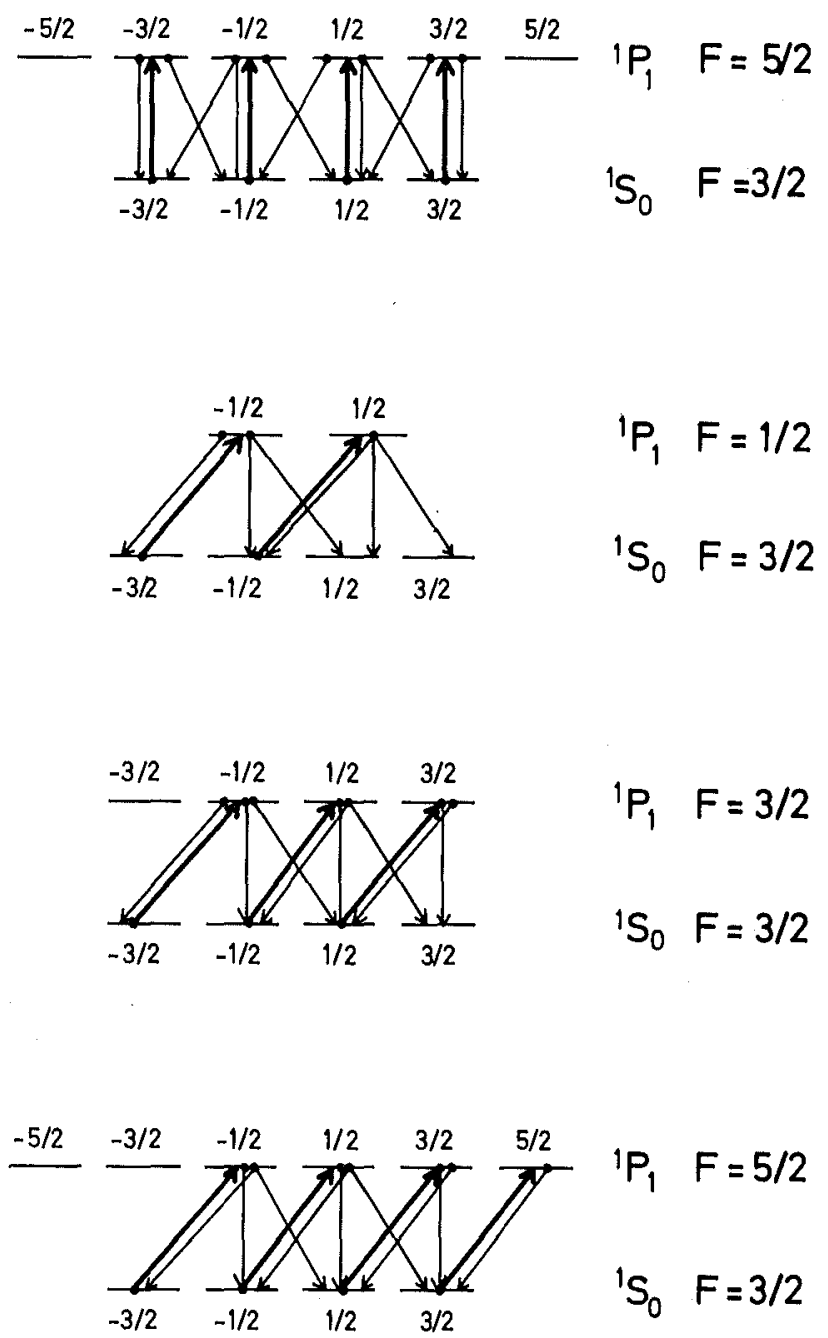

Fig. A2:

Optical pumping effects with $\sigma$-light in a ${ }^{1} \mathrm{~S}_{0}-{ }^{1} \mathrm{P}_{1}$ transition for a $I=3 / 2$ nucleus. 